\title{
Development of a Lateral-directional Envelop Protection Flight Control System
}

\author{
Liu Fan, Wang Lixin \\ School of Aeronautic Science and Engineering \\ Beihang University, \\ Beijing 100191, China
}

\begin{abstract}
For the factor of aircraft flying safety, some critical flight parameters must be limited within the envelop borders. However, excellent flight performance requires that the aircraft can flight to some envelop borders easily and fast nearly. The purpose of envelop protection is to solve this dilemma. Based on the basic lateral-directional flight control law, an envelop protection method using control command compensation is developed. Numerical simulation results show that this method can effectively solves the flying safety problem without sacrificing flight performance.
\end{abstract}

Keywords- Aircraft, Envelop protection, Envelop limit, Command compensation, Flight control system, Lateraldirectional control, Simulation

\section{INTRODUCTION}

Due to the factors of controllability, aircraft structure endurance and comfortablity of passenger and crew, critical flight parameters of the aircraft should not excess their borders, e.g. bank angle and sideslip angle. However, the best flight performances require flight to reach some envelop borders easily and fast nearly. Therefore, its flight control system must adopt envelop protection system to avoid risks when pilots try to reach envelop borders, and ensure pilot convenience manipulation.

\section{TYPE STYLE AND FONTS}

The lateral-directional envelop protection consists of bank angle protection and sideslip angle protection, which is an assistant function of the basic flight control law, as illustrated in Figure1.

In this system, Order shaping converts pilot forces into flight commands. Then the basic flight control law executes the track of these commands. The envelop protectors compensate the commands when flight parameters are about to exceeding their borders.

The principle of envelop protection design is as follows. For the rolling axis, the command is $\phi_{\mathrm{c}}$, which is defined as the change rate of bank angle. The flight will hold the bank angle when $\phi_{\mathrm{c}}$ recurs to 0 . Therefore, bank angle protection requires $\phi_{\mathrm{c}}$ to fast decrease to 0 when bank angle is near its border, as a result, bank angle stops increasing immediately. For the yawing axis, both command and protect target is sideslip angle, therefore sideslip angle protection requires the command to keep being less than its border directly[1].

According to the principle of envelop protection, the envelop protector control law can be presented as follows.

$$
\begin{aligned}
& \dot{\phi}_{b}=\left\{\begin{array}{lc}
k_{\phi} \cdot\left(\phi+k_{\dot{\phi}} \cdot \dot{\phi}\right) & \left|\phi+k_{\dot{\phi}} \cdot \dot{\phi}\right|>\phi_{\lim }-\Delta_{\phi} \\
0 & \text { else }
\end{array}\right. \\
& \beta_{b}= \begin{cases}k_{\beta} \cdot\left(\beta+k_{r} \cdot r\right) & \left|\beta+k_{r} \cdot r\right|>\beta_{\lim }-\Delta_{\beta} \\
0 & \text { else }\end{cases}
\end{aligned}
$$

where $\dot{\phi}_{b}$ and $\beta_{b}$ are the compensational commands, which amend respectively the pilot commands $\dot{\phi}_{b}$ and $\beta_{b}$. The suffix "Lim" denotes the border, and $\Delta$ denotes safety margin. $\kappa_{\phi}, \kappa_{\beta}, \kappa_{\dot{\phi}}$ and $\kappa_{\gamma}$ are the gains used for the adjustment of protection effect.

Formula(1) indicates, $\kappa_{\beta}$ and $\kappa_{\phi}$ directly affect the values of compensational commands. The higher $\kappa_{\beta}$ and $\kappa_{\phi}$ are, the more easily bank angle and sideslip angle protections will be limited. However, excessive $\kappa_{\beta}$ and $\kappa_{\phi}$ will cause oscillation of flight states. $\kappa_{\dot{\phi}}$ and $\kappa_{\gamma}$ determine the performance of anticipation for compensation. Similarly, high $\kappa_{\dot{\phi}}$ and $\kappa_{\gamma}$ are beneficial to realize envelop protection. However, excessive $\kappa_{\beta}$ and $\kappa_{\phi}$ will onset command compensation at samll bank angle and sideslip angle, which leads to that the flight can't reach the envelop borders, as a result, holdbacks the best exertion of the flight performances.

\section{FLIGHT LATERAL-DIRECTIONAL CLOSED- LOOP CONTROL SYSTEM}

As illustrated in Figure1, besides envelop protector, flight lateral-directional closed-loop control system mainly comprises flight lateral-directional dynamics model and basic flight lateral-directional flight control law.

\section{A. Flight lateral-directional dynamics model[2]}

For the aircraft dynamics, it is assumed that: the aircraft is in a straight and level flight. Small perturbation equation of flight lateral-directional linear dynamics model can be presented as follows.

$$
\begin{gathered}
\dot{x}=A x+B u \\
x=\left[\begin{array}{c}
\beta \\
p \\
r \\
\phi
\end{array}\right] \quad \mathrm{u}=\left[\begin{array}{c}
\delta_{a} \\
\delta_{r}
\end{array}\right] \quad A=\left[\begin{array}{llll}
\bar{Y}_{\beta} & \alpha_{*}+\bar{Y}_{p} & \bar{Y}_{r}-1 & g \cos \theta_{*} / V_{*} \\
\bar{L}_{\beta} & \bar{L}_{p} & \bar{L}_{r} & 0 \\
\bar{N}_{\beta} & \bar{N}_{p} & \bar{N}_{r} & 0 \\
0 & 1 & \tan \theta_{*} & 0
\end{array}\right]
\end{gathered}
$$




$$
B=\left[\begin{array}{rl}
0 & \bar{Y}_{\delta_{r}} \\
\bar{L}_{\delta_{a}} & \bar{L}_{\delta_{r}} \\
\bar{N}_{\delta_{s}} & \bar{N}_{\delta_{r}} \\
0 & 0
\end{array}\right]
$$

where $\beta, p, r, \phi$ denote disturbances about reference motion, $\delta_{a}$ and $\delta_{r}$ denote respectively aileron and rudder input. $V_{*}, \theta_{*}$ and $D_{*}$ in formula (5) denote the reference longitudinal states. Amendment force and moment derivates in formula(3) are presented as follows.

$$
\left.\begin{array}{lr}
\bar{L}_{i}=\frac{L_{i}+\left(I_{z x} / I_{z}\right) N_{i}}{I x-I_{z x}^{2} / I z} & \\
\bar{N}_{i}=\frac{N_{i}+\left(I_{z x} / I_{z}\right) L_{i}}{I_{z}-I_{z x}^{2} / I_{x}} & \left(i=\beta, p, r, \delta_{a}, \delta_{r}\right)
\end{array}\right\}
$$

where $L_{i}, N_{i}$ and $C_{i}$ denote respectively rolling moment derivate, yawing moment derivate and side force derivate. $I_{x}$ and $I_{z}$ denote respectively moment of inertia in roll and yaw. $m$ denotes mass aircraft. $I_{z x}$ denotes product of inertia about $o x$ and $o z$ axis.

\section{B. Lateral-directional basic flight control law [3]}

The structure of the basic lateral-directional flight control law is illustrated in Figure2.

In the rolling axis, inner loop rate $\dot{\phi}$ feedback improves damping of roll model. Bases on this, outer loop rate $\dot{\phi}$ feedback and PI controller compose SCAS of rolling axis, which enables the flight responses track the command $\dot{\phi}_{c}+\dot{\phi}_{b}$. In the yawing axis, inner loop rate $r$ and $\beta$ feedback improve yawing modal characteristic, outer loop $\beta$ feedback and PI controller compose SCAS of yawing axis, which enables the flight responses track the command $\beta_{c}+\beta_{b}$.

Bases on the flight dynamics model and the basic flight control law, flight closed loop control system can be expressed with equation in matrix presented as follows.

where $\beta, p, r$ and $\phi$ are the flight responses. $\dot{\phi}_{c}+\dot{\phi}_{b}$ and $\beta_{c}+\beta_{b}$ denote the control commands compensated by envelop protectors. $\varepsilon_{\dot{\phi}}, \mathcal{E}_{\beta}$ and $\varepsilon_{f}$ are the states of control system. $\delta_{a}$ and $\delta_{r}$ denote respectively aileron and rudder input. $K_{p_{-} \dot{\phi}}, K_{\mathrm{I}_{-} \dot{\phi}}, K_{\dot{\phi}}, K_{P_{-} \beta}, K_{i_{-} \beta}, K_{r}, \mathcal{\varepsilon}_{\dot{\phi}}, \varepsilon_{\beta}, \tau_{a}, \tau_{r}, \tau_{w}$ and $r_{f}$ are as illustrated in Figure2. A and B are presented in Formula (3).

\section{SIMULATION AND ANALYSIS}

The simulation is implemented using flight lateraldirectional closed-loop control system which includes envelop protectors. It starts at an initial condition of altitude $\mathrm{H}=3000 \mathrm{~m}$, velocity $\mathrm{V}=160 \mathrm{~m} / \mathrm{s}$, in straight and level flight. Simulation curves are shown in Figure.3-4.

As Figure 3 indicates, before bank angle reachs its borde $\phi_{\text {lim }}$, flight response of $\dot{\phi}$ follows the command $\dot{\phi}_{c}$, and bank angle keeps increasing. Without protection, bank angle $\phi$ excesses its border very soon, while $\dot{\phi}$ decreases to 0 and bank angle $\phi$ is limited under its border because of the compensational command $\dot{\phi}_{b}$ when bank angle protection is implemented. Simulation curves in Figure 4 also demonstrates sideslip angle protection can prevents sideslip angle from excessing its border $\beta_{\text {lim }}$.

Simulation result reflects the principle of envelop protection and satisfying effect.

\section{SUMMARIES}

Based on the lateral-directional basic flight control law, an lateral-directional envelop protection flight control system which adopt the mothed of control command compensation is designed. Simulation result demonstrates that the envelop protection system can effectively limits the protect targets under their borders.

\section{REFERENCES}

[1] Rudolf Brockhaus. Flugregelung[M], 1999, PP: 498-500

[2] FANG Zhen-pin; CHEN Wan-chun; ZHANG Shu-guang. Flight dynamics of Aircraft. Publishing company of Beihang University [M], 2005, PP: 202-203.

[3] F. Holzapfel; O. da Costa; M. Heller; G. Sachs. Development of a Lateral-Directional Flight Control System for a New Transport Aircraft. AIAA Guidance, Navigation, and Control Conference, Keystone, CO [C], 2006. 

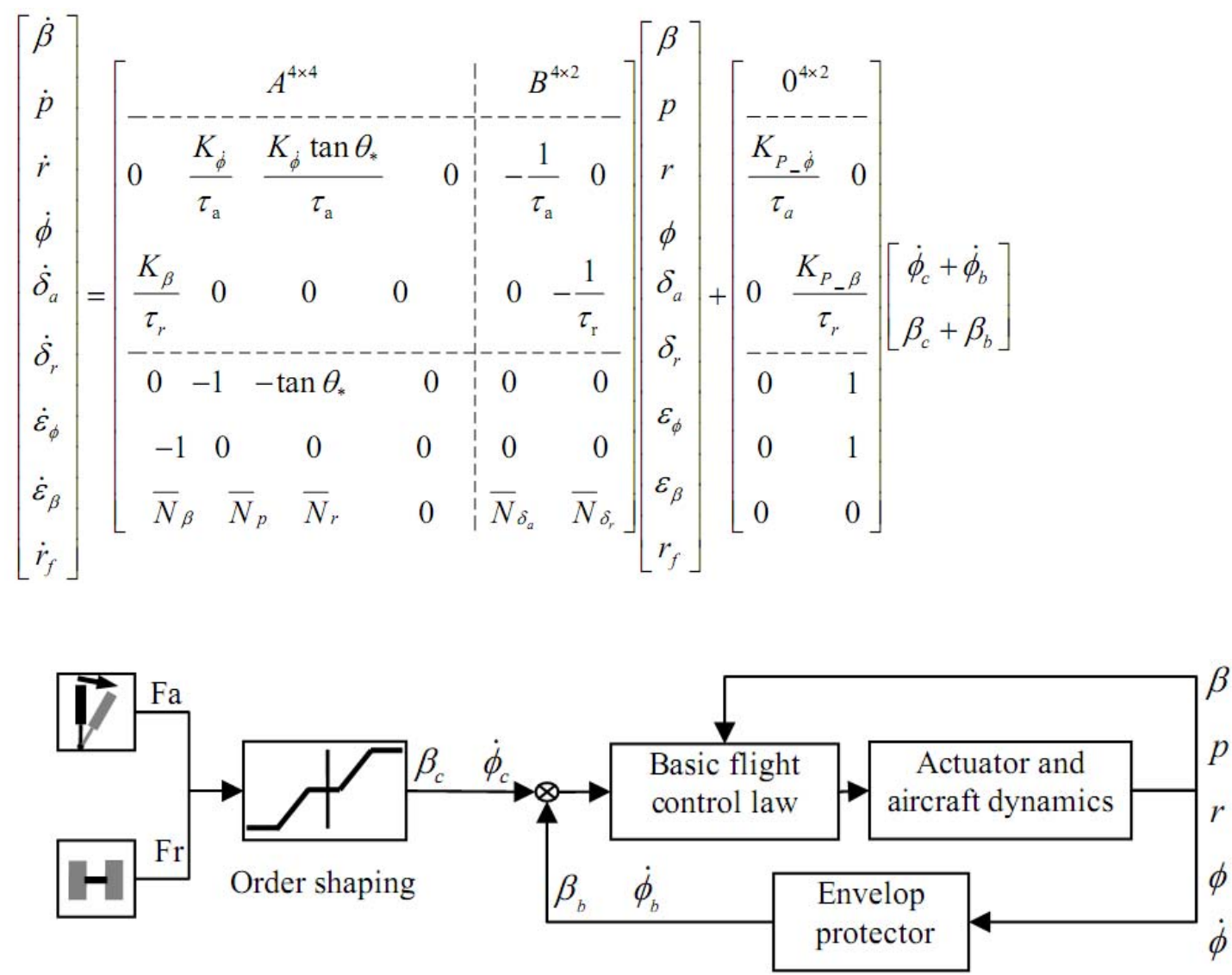

Figure 1. The lateral-directional flight control system

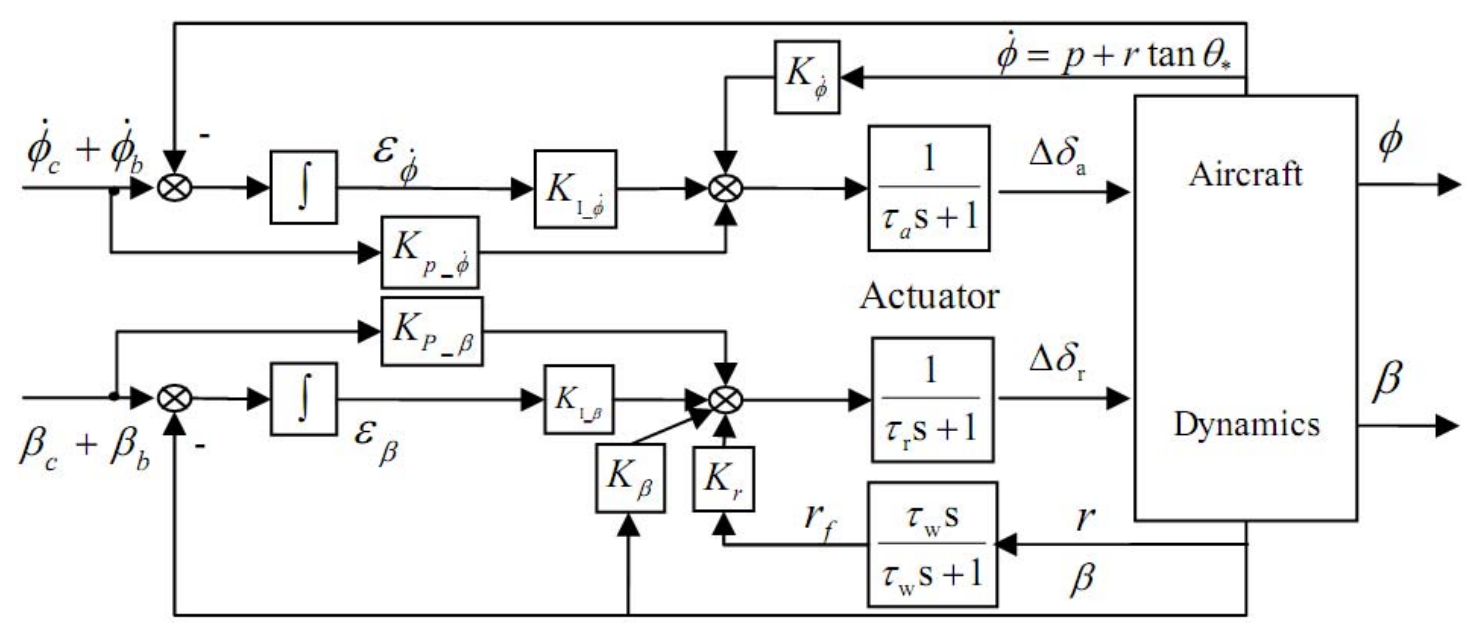

Figure 2. The Layout Lateral-Directional Basic Flight Control System 

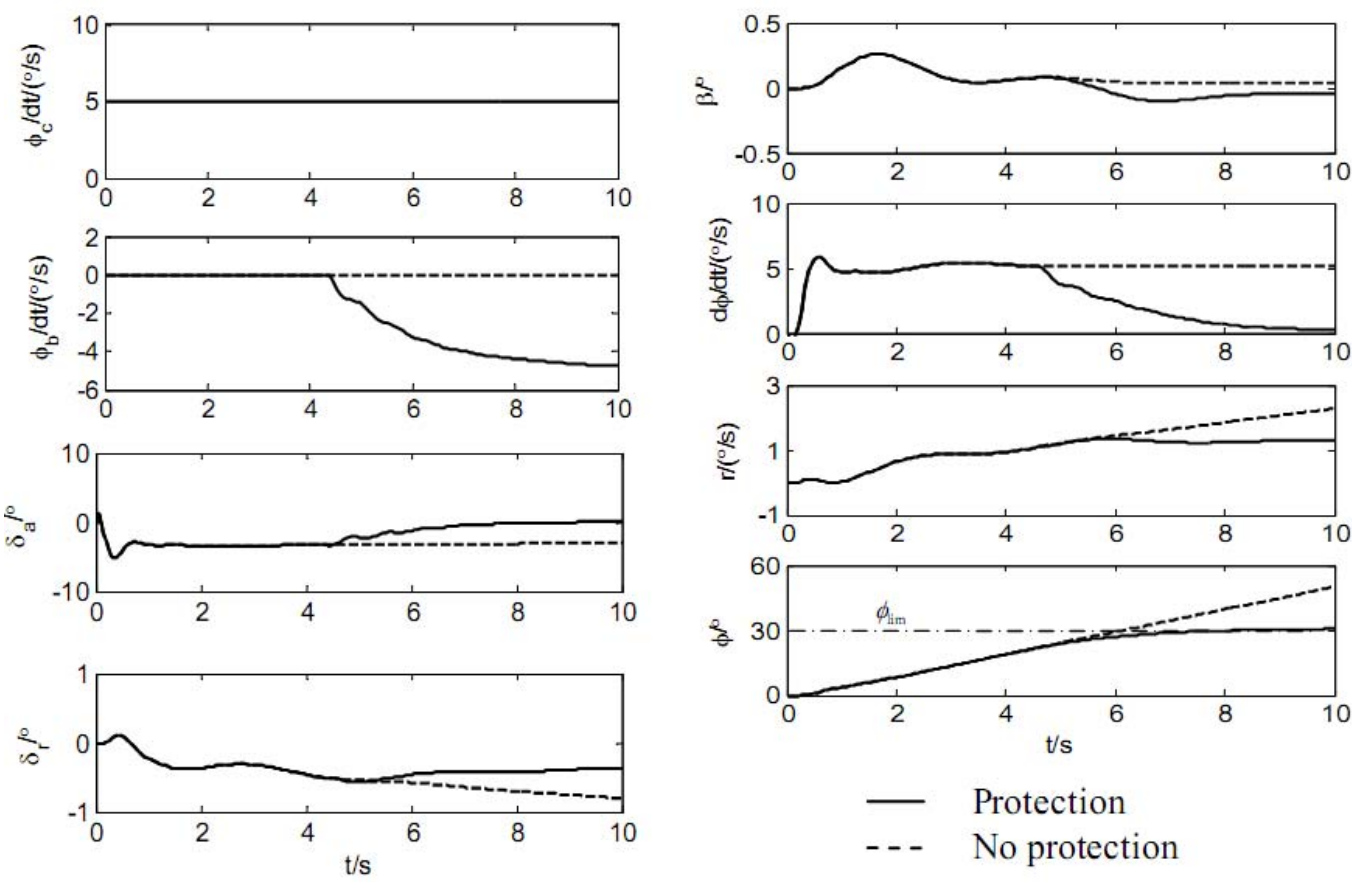

Figure 3. Bank angle protection simulation
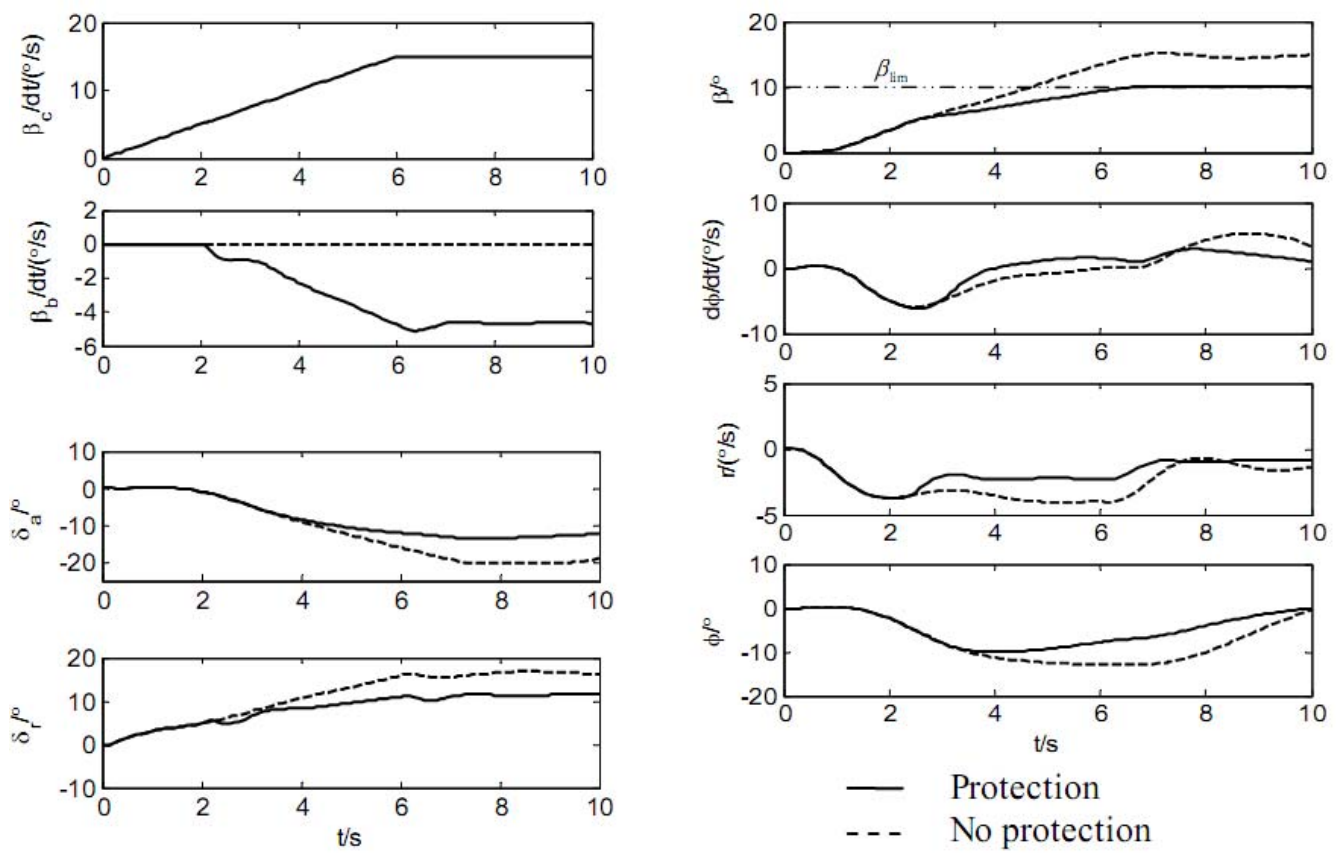

Figure 4. Sideslip angle protection simulation 